
melão pele-de-sapo em duas densidades de plantio. Horticultura Brasileira 26: 236-239.

\title{
Produtividade e qualidade de frutos de melão pele-de-sapo em duas den- sidades de plantio
}

\author{
Glauber Henrique de S Nunes; Elaíne WL Pereira; Rui Sales Júnior; Francisco Bezerra Neto; Kelly \\ Cristina de Oliveira; Luciene X Mesquita \\ ${ }^{1}$ UFERSA, C. Postal 137, 59625-900 Mossoró-RN; glauber@ufersa.edu.br
}

\begin{abstract}
RESUMO
Existem dúvidas se a densidade utilizada no melão amarelo permite a colheita de frutos do tipo pele-de-sapo adequados para o mercado externo. Dois experimentos foram instalados com o objetivo de avaliar híbridos de melão do tipo pele-de-sapo, em duas densidades de plantio, 10 e 15 mil plantas $\mathrm{ha}^{-1}$. No primeiro experimento foram avaliados os híbridos Sancho, PS 07 e Tendency, e, no segundo, os híbridos PS RDR, PS 10, PS 15, PS 21 e PS 32. Utilizou-se delineamento em blocos ao acaso, com quatro repetições. Os tratamentos foram arranjados em parcelas subdivididas, com o fator densidade disposto na parcela e, o fator híbrido, na sub-parcela. As subparcelas foram compostas por 16 e 24 plantas na área útil, no primeiro e no segundo experimentos, respectivamente. Não houve interação significativa entre híbridos e densidade de plantio. A produtividade e número total de frutos na densidade de plantio de 15.000 plantas ha ${ }^{-1}$ foram maiores, com valores médios de 38,95 $\mathrm{t} \mathrm{ha}^{-1} \mathrm{e}$ 23,05 mil frutos $\mathrm{ha}^{-1}$, respectivamente. A firmeza da polpa e o teor de sólidos solúveis totais não foram influenciados pela densidade de plantio. Por sua vez, os híbridos diferiram para as características número total de frutos, produtividade, firmeza da polpa e teor de sólidos solúveis totais. Os híbridos Sancho e Tendency, com boa produtividade (31,3 e 32,9 $\mathrm{t} \mathrm{ha}^{-1}$, respectivamente) e frutos de tamanho superior a $3,0 \mathrm{~kg}$, com boa firmeza ( 28 e $27 \mathrm{~N}$, respectivamente) e teor de sólidos $(9,33$ e $12,03 \%)$ acima do limite aceito para comercialização, se sobressaíram no experimento I; enquanto os híbridos PS 15 e PS 32 se destacaram, pelas mesmas características (respectivamente, 36,6 e 41,3 $\mathrm{t} \mathrm{ha}^{-1}$; frutos com 3,10 e 3,02 kg, com firmeza de 27 e $28 \mathrm{~N}$ e porcentagem de sólidos solúveis de 12,03 e $9,96 \%$ ), no segundo experimento. Ressalta-se, porém, que o híbrido PS 32 apresentou um maior número de frutos $\left(25,2 \mathrm{mil} \mathrm{ha}^{-1}\right)$ que o híbrido PS 15 (19,7), embora este tenha sido o material cujos frutos apresentaram a maior porcentagem de sólidos totais.
\end{abstract}

Palavras-chave: Cucumis melo, competição de cultivares, híbridos, manejo cultural.

\author{
ABSTRACT \\ Fruit yield and quality of Piel de Sapo melon in two planting \\ densities
}

There are doubts whether the planting density used for Yellow melon allows harvesting Piel del Sapo fruits that meet the standards of foreign markets. Two experiments were carried out to evaluate Piel de Sapo hybrids in two planting densities, 10 and 15 thousand plants ha ${ }^{-1}$. Hybrids Sancho, PS 07, and Tendency were evaluated in the first experiment, while hybrids PS RDR, PS 10, PS 15, PS 21, and PS 32 were evaluated in the second experiment. The experimental design used was blocks at random, with four replications. Treatments were arranged in split-plot, with planting densities assigned to plots and hybrids to sub-plots. Sub-plots had 16 and 24 plants in the useful area in the first and the second experiments, respectively. There was no significant interaction between planting densities and hybrids in none of the assessed traits. Fruit yield and total number were significantly higher in the planting density of 15,000 plants $\mathrm{ha}^{-1}$, with mean values of $38.95 \mathrm{t} \mathrm{ha}^{-1}$ and 23.05 thousand fruits ha-1, respectively, in comparison to 10,000 plants ha ${ }^{-1}$. Pulp firmness and total content of soluble solids were not influenced by planting density. There were significant differences among hybrids for fruit total number, yield, pulp firmness and total content of soluble solids. In the first experiment, hybrids Sancho and Tendency had the best performance, with fruit yields of respectively 31.3 and $32.9 \mathrm{t} \mathrm{ha}^{-1}$, with average weight over than $3.0 \mathrm{~kg}$, pulp firmness of 28 and $27 \mathrm{~N}$, respectively, and soluble solids contents of 9.33 and $12.03 \%$, therefore above the market limit. In the second experiment, hybrids PS 15 and PS 32 outstood, with yields of 36.6 and $41.3 \mathrm{t} \mathrm{ha}^{-1}$, respectively, fruit weight of 3.10 and $3.02 \mathrm{~kg}$, pulp firmness of 27 and $28 \mathrm{~N}$, respectively, and soluble solids contents of 12.03 and $9.96 \%$. Worth mentioning that hybrid PS 32 produced significantly more fruits (25.2 thousand fruits $\left.\mathrm{ha}^{-1}\right)$ as compared to hybrid PS 15 (19.7 thousand fruits $\mathrm{ha}^{-1}$ ).

Keywords: Cucumis melo, cultivar evaluation, hybrids, crop practices.

\section{(Recebido para publicação em 15 de janeiro de 2007; aceito em 29 de maio de 2008)}

$\mathrm{O}$ cultivo do melão é a principal atividade do agronegócio no Rio Grande do Norte. O Estado potiguar é o maior produtor e exportador brasileiro dessa cucurbitácea. Em 2003, o Brasil produziu $349.498 \mathrm{t}$, sendo o estado responsável por 192.421 t, o que correspondeu a $55,1 \%$ da produção nacional (IBGE, 2004).

Embora a maior parte do melão produzido no estado seja do tipo amarelo, nos últimos anos tem aumentado a área de cultivo do melão pele-de-sapo, em razão da boa cotação do produto na Europa. Sales Júnior et al. (2004) relatam que aproximadamente $11 \%$ dos frutos de melão exportados pelo porto de Natal em 2002 pertenciam ao tipo pele-desapo. Com a crescente demanda do mercado exterior, a tendência é o aumento da área de cultivo com o melão pelede-sapo.
$\mathrm{O}$ mercado europeu exige frutos de melão amarelo com pesos entre 1,5 e 1,8 $\mathrm{kg}$. No caso do melão pele-de-sapo, a exigência é por frutos grandes, em torno de $3,5 \mathrm{~kg}$, em especial no mercado espanhol. As práticas de cultivo utilizadas no Agropolo Mossoró-Assu estão bem definidas para o melão amarelo. Por outro lado, existem poucos artigos na literatura sobre as práticas de cultivo com o melão tipo pele-de-sapo. Com 
relação à densidade de semeadura, por exemplo, os artigos contemplam, principalmente, o melão amarelo e o cantaloupe (Paris et al., 1988; Grangeiro et al., 1999a, 1999b; Silva et al., 2003; Chaves et al. 2004). Existem dúvidas, por parte dos produtores, se a densidade utilizada no melão amarelo vai permitir a colheita de frutos do tipo pelede-sapo com o tamanho comercial exigido pelo mercado externo.

Ainda há carência de informações sobre as cultivares recomendadas para a região, com poucas opções disponíveis para os produtores. Além disso, as cultivares atualmente utilizadas não foram avaliadas nas diversas condições de cultivo do pólo agrícola Mossoró-Assú. Considerando que a cada ano são lançados muitos híbridos no mercado, é fundamental que os mesmos sejam avaliados in loco, uma vez que a adoção de genótipos sem uma avaliação prévia pode acarretar prejuízos na produtividade e qualidade do produto (Nunes et al., 2004). O objetivo do presente trabalho foi avaliar a produtividade e qualidade de frutos de melão pele-de-sapo em duas densidades de semeadura.

\section{MATERIAL E MÉTODOS}

O estudo foi realizado no município de Mossoró (RN) no ano agrícola de 2001. O clima da região, segundo a classificação de Koppen, é BSWh ou muito seco, com estação de chuva no verão atrasando-se para o outono (Carmo Filho \& Oliveira, 1989).

Dois experimentos foram instalados no mês de julho, em áreas contíguas, na Horta da Universidade Federal Rural do Semi-árido (UFERSA), para avaliar híbridos de melão do tipo pele-de-sapo em duas densidades de plantio. No experimento I, foram avaliados os híbridos Sancho, PS 07 e Tendency, e, no experimento II, os híbridos PS RDR, PS 10, PS 15, PS 21 e PS 32. Todos os híbridos têm expressão sexual andromonóica e possuem frutos oblongos, com casca verde e polpa branca. As densidades de plantio utilizadas foram 10.000 e 15.000 plantas ha- ${ }^{-1}$.

O preparo do solo constou de uma aração e uma gradagem, seguido de sulcamento em linhas espaçadas de $2 \mathrm{~m}$, com profundidade de aproximadamente $20 \mathrm{~cm}$. A adubação de fundação foi realizada em sulcos, nas seguintes dosagens: esterco bovino $=12 \mathrm{t} \mathrm{ha}^{-1}$, formulação $(6-24-12)=600 \mathrm{~kg} \mathrm{ha}^{-1}$, superfosfato simples $=160 \mathrm{~kg} \mathrm{ha}^{-1} \mathrm{e}$ calcário $=90 \mathrm{~kg} \mathrm{ha}^{-1}$. Os adubos foram incorporados com enxada rotativa. As adubações de cobertura foram realizadas em fertirrigação, diariamente, seguindo a necessidade da cultura. As quantidades totais utilizadas no final do ciclo foram: nitrato de amônio $=250 \mathrm{~kg}$ $\mathrm{ha}^{-1}$, cloreto de potássio $=450 \mathrm{~kg} \mathrm{ha}^{-1}$, uréia $=90 \mathrm{~kg} \mathrm{ha}^{-1}$ e ácido fosfórico $=90$ $\mathrm{L} \mathrm{ha}^{-1}$. As adubações foliares constaram de aplicações de $20 \mathrm{~L} \mathrm{ha}^{-1}$ da formulação $8 \% \mathrm{Ca}$ e $2 \%$ B. Foram realizadas capinas manuais até 30 dias após o transplantio. O controle fitossanitário foi feito aplicando-se inseticida semanalmente, até 35 dias após o transplantio para combater a mosca-branca (Nunes et al., 2005). Os frutos foram colhidos manualmente com o auxílio de canivete, colocados em caixas plásticas e enviados para o Laboratório de Química e Pós-colheita da UFERSA.

Utilizou-se o delineamento em blocos casualizados com quatro repetições. No primeiro experimento, a parcela foi composta por doze linhas de cinco metros e, no segundo, por quinze linhas de cinco metros. Os tratamentos foram arranjados em parcelas subdivididas, com o fator densidade nas parcelas e, o fator híbrido, nas sub-parcelas. A subparcela, no primeiro experimento, foi formada por quatro linhas, com as duas fileiras centrais correspondendo à área útil. No segundo experimento, a área útil da subparcela foi composta por três fileiras.

Foram avaliados: a) número total de frutos (contagem do número de frutos da área útil da parcela); b) peso médio dos frutos (média do peso de 20 frutos por parcela, avaliado em balança de precisão); c) produtividade (pesagem e contagem dos frutos produzidos); d) firmeza da polpa [o fruto foi dividido longitudinalmente e em cada parte foi medida a resistência, utilizando um penetrômetro com pluger de ponta cônica de $8 \mathrm{~mm}$ de diâmetro, aplicado na região mediana comestível de cada parte do fruto (duas leituras por fruto em regiões diferentes), eqüidistante em relação ao comprimento e à espessura da polpa]; e) teor de sólidos solúveis [através de refratometria digital com compensação de temperatura automática (escala de 0 a $32 \%$ ), pela amostragem de uma fatia de 20 frutos por parcela, efetuando-se duas leituras por fruto].

Os dados foram submetidos à análise de variância, com posterior aplicação do teste de Scott-Knott a 5\% de probabilidade para o agrupamento de média dos híbridos e teste t para densidade de plantio. Para a realização das análises estatísticas foi utilizado o programa SISVAR (Ferreira, 2000).

\section{RESULTADOS E DISCUSSÃO}

Não se observou interação significativa entre os fatores densidade de plantio e híbrido, indicando comportamento consistente dos híbridos nas duas densidades de plantio avaliadas. Resultado semelhante também foi observado por Chaves et al. (2004), estudando a combinação de dois híbridos de melão (Honey Dew e Hy Mark) e quatro densidades de plantio $(20,30,40$ e 50 mil plantas ha-1).

Nos dois experimentos, observou-se efeito da densidade de plantio apenas para as características número total de frutos e produtividade (Tabela 1). O número total de frutos por ha foi maior na densidade de plantio de 15 mil plantas ha-1, sem contudo ser observada redução significativa no peso médio dos frutos em função do aumento da densidade de plantio (Tabela 1). Avaliando a influência da densidade de plantio sobre híbridos de melão amarelo em Mossoró, Grangeiro et al. (1999a) observaram que o aumento da densidade proporcionou maior número de frutos por hectare para os híbridos Gold Mine, AF-646 e XPH 13096. Silva et al. (2003), também na região de Mossoró, verificaram aumento do número de frutos do híbrido Gold Pride em densidades maiores.

Em conseqüência, a produtividade foi maior no plantio mais adensado (Tabela 1), confirmando os resultados obtidos por Grangeiro et al. (1999a), Resende \& Costa (2003) e Silva et al. (2003). Não obstante, Pereira et al. 
Tabela 1. Número total, peso médio e produtividade de frutos de melão tipo pele-de-sapo em duas densidades de plantio (fruit total number, average weight, and yield of piel de sapo melon, in two planting densities). Mossoró, UFERSA, 2001.

\begin{tabular}{|c|c|c|c|c|}
\hline Experimentos & $\begin{array}{l}\text { Densidade } \\
\left(\text { plantas ha }{ }^{-1}\right)\end{array}$ & $\operatorname{NTF}^{1}\left(m^{\prime l ~ h a-1}\right)$ & $\mathrm{PM}^{2}(\mathrm{~kg})$ & $\operatorname{PROD}^{3}\left(\mathrm{t} \mathrm{ha}^{-1}\right)$ \\
\hline \multirow{2}{*}{ I } & 10.000 & $17,9 b$ & $3,01 \mathrm{a}$ & $35,3 b$ \\
\hline & 15.000 & $23,1 \mathrm{a}$ & $2,91 \mathrm{a}$ & $39,5^{\mathrm{a}}$ \\
\hline \multirow[b]{2}{*}{ II } & 10.000 & $18,3 b$ & $3,00 a$ & $32,9 b$ \\
\hline & 15.000 & $23,0 a$ & $2,87 a$ & $38,6^{a}$ \\
\hline
\end{tabular}

Médias seguidas de mesma letra nas colunas, em cada experimento, não diferem entre si pelo teste de Scott-Knott, $\mathrm{p}<0,05$ (Means followed by the same letter in the column, in each experiment, do not differ from each other by the Scott-Knott test, $\mathrm{p}<0.05) .{ }^{1} / \mathrm{NTF}=$ número total de frutos (fruit total number); ${ }^{2} / \mathrm{PM}=$ peso médio do fruto (fruit average weight); ${ }^{3 /}$ PROD $=$ produtividade (yield).

Tabela 2. Número total, peso médio, firmeza da polpa e teor de sólidos solúveis de frutos de melão pele-de-sapo, em duas densidades de plantio (Fruit total number, average weight, yield, pulp firmness, and content of soluble solids of piel de sapo melon, in two planting densities). Mossoró, UFERSA, 2001.

\begin{tabular}{|c|c|c|c|c|c|c|}
\hline Experimento & Híbrido & $\begin{array}{c}\mathrm{NFT}^{1} \\
\left(\mathrm{mil} \mathrm{ha}^{-1}\right)\end{array}$ & $\begin{array}{l}\mathrm{PM}^{2} \\
(\mathrm{~kg})\end{array}$ & $\begin{array}{l}\text { PROD }^{3} \\
\text { (t ha-1) }\end{array}$ & $\begin{array}{l}\text { FP }^{4} \\
(\mathrm{~N})\end{array}$ & $\begin{array}{c}\text { SST }^{5} \\
(\%)\end{array}$ \\
\hline & Sancho & $20,6 a$ & $3,02 a$ & $31,3 a$ & $28 a$ & $9,33 b$ \\
\hline \multirow[t]{4}{*}{ I } & PS 07 & $17,6 b$ & $2,76 a$ & $26,0 b$ & $22 b$ & $9,93 \mathrm{~b}$ \\
\hline & Tendency & $23,3 a$ & $3,10 a$ & $32,9 a$ & $27 a$ & $12,03 a$ \\
\hline & PS RDR & $18,8 b$ & $2,88 a$ & $31,2 b$ & $29 a$ & $8,93 b$ \\
\hline & PS 10 & $18,0 \mathrm{~b}$ & $2,91 \mathrm{a}$ & $25,6 c$ & $26 a$ & $11,68 a$ \\
\hline \multirow[t]{3}{*}{ II } & PS 15 & $19,7 b$ & $3,10 a$ & $36,6 a$ & $27 a$ & $12,03 a$ \\
\hline & PS 21 & $20,7 b$ & $2,76 a$ & $40,0 a$ & $22 b$ & $9,93 b$ \\
\hline & PS 32 & $25,2 a$ & $3,02 a$ & $41,3 a$ & $28 a$ & $9,96 b$ \\
\hline
\end{tabular}

Médias seguidas de mesma letra nas colunas, em cada experimento, não diferem entre si pelo teste de Scott-Knott, $\mathrm{p}<0,05$ (Means followed by the same letter in the column, in each experiment, do not differ from each other by the Scott-Knott test, $\mathrm{p}<0.05)$. ${ }^{1} / \mathrm{NTF}=$ número total de frutos (fruit total number); ${ }^{2} / \mathrm{PM}=$ peso médio do fruto (fruit average weight); ${ }^{3} /$ $\mathrm{PROD}=$ produtividade $($ yield $) ;{ }^{4} / \mathrm{FP}=$ firmeza da polpa (pulp firmness); $5 / \mathrm{SST}=$ teor de sólidos solúveis (content of soluble solids).

(2003) e Chaves et al. (2004) observaram decréscimo na produtividade com o aumento da densidade. Deve ser ressaltado que, dependendo da cultivar, o aumento de produtividade é crescente até determinada população de plantas, a partir da qual há um decréscimo na produtividade. Isso ocorre em razão da competição excessiva entre as plantas por água, nutrientes e radiação solar, devido o aumento do índice de área foliar. O índice de área foliar aumenta com o desenvolvimento da planta e com o número de plantas na área, variando em função das condições edafo-climáticas (Holiday, 1969). O aumento da produtividade deveu-se ao maior número de frutos produzidos, uma vez que o peso médio do fruto não diferiu nas duas densidades. Robinson \& Decker-Walters (1997) comentam que em cucurbitáceas a produtividade depende tanto do número total de frutos como o tamanho do fruto e que o adensamento da cultura proporciona maior número de frutos e menor peso médio, devido às pressões de competição entre plantas.

Os resultados obtidos com os híbridos foram bastante satisfatórios para a região. A produtividade de meloeiro no Nordeste tem um intervalo de variação entre 17 e 30 t ha $^{-1}$ (Dias et al., 1998). No caso do melão pele-de-sapo, Nunes et al (2005) verificaram produtividades entre 27 e 52,4 tha ${ }^{-1}$, com média de 38,0 t ha ${ }^{-1}$. No presente trabalho, nos dois experimentos, a média dos híbridos foi superior a $30 \mathrm{t} \mathrm{ha}^{-1}$, com destaque para os híbridos Sancho e Tendency, no experimento I, e PS 15, PS 21 e PS 32, no experimento II (Tabela 2). No experimento I, observou-se que os híbridos
Sancho e Tendency produziram maior número de frutos que o híbrido PS 07, enquanto no experimento II sobressaiuse o híbrido PS 32 (Tabela 2).

Dos aspectos internos do fruto, a firmeza da polpa é essencial, uma vez que é uma das características que determinam o tempo de vida útil pós-colheita de uma cultivar (Menezes et al., 2001). A experiência tem mostrado que frutos de melão do tipo pele-de-sapo quando colhidos com firmeza de polpa na faixa de 26 a $28 \mathrm{~N}$ chegam às prateleiras européias com boa conservação pós-colheita. Com efeito, pode-se inferir, a partir das estimativas das médias de firmeza da polpa do presente trabalho, que apenas os frutos dos híbridos PS 07 (experimento I) PS 21 (experimento II) não estariam adequados para exportação (Tabela 2).

O teor de sólidos solúveis tem sido apontado como a característica mais importante da qualidade de fruto de melão (Artés et al., 1993). Segundo Alves et al. (2000), o teor de sólidos solúveis totais recomendado para o melão pele-de-sapo é de $11 \%$. Todavia, na prática tem sido adotado o valor mínimo de $9,0 \%$ para comercialização dos vários tipos de melão (Sales Júnior $e t$ al., 2004). No presente trabalho, o híbrido Tendency foi o de maior destaque para essa característica no experimento I, enquanto os híbridos PS 10 e PS 15 se destacaram no experimento II. A maioria dos híbridos apresentou estimativas do teor de sólidos totais próximas do valor mínimo $(9,0 \%)$, com exceção do híbrido PS RDR, embora não tenha diferido significativamente dos demais híbridos incluídos em seu grupo (Tabela 2). É possível afirmar, portanto que, em relação ao teor de sólidos totais, todos os híbridos avaliados poderiam ser comercializados no mercado externo.

De modo geral, os híbridos Sancho e Tendency, com boa produtividade e frutos de tamanho superior a $3,0 \mathrm{~kg}$, com boa firmeza e teor de sólidos acima do limite aceito para comercialização, se sobressaíram no experimento I; enquanto os híbridos PS 15 e PS 32 se destacaram, pelas mesmas características, no segundo experimento. Ressalta-se, porém, que o híbrido PS 32 apresentou um maior número de frutos que o híbrido 
PS 15, embora este tenha sido o material cujos frutos apresentaram a maior porcentagem de sólidos totais. Pelos resultados obtidos, recomenda-se a densidade de plantio de 15.000 plantas ha-1.

\section{REFERÊNCIAS}

ALVES RE; PIMENTEL CR; MAIA CE; CASTRO EB; VIANA FM; COSTA FV; ANDRADE GG; FILGUEIRAS HAC; ALMEIDA JHS; MENEZES JB; COSTA JG; PEREIRA LSE. 2000. Manual de melão para exportação. Brasília: Embrapa. 51p.

ARTÉS F; ESCRICHE AJ; MARTINEZ JA; MARIN JG.1993. Quality factors in four varieties of melon (Cucumis melo L.). Journal of Food Quality 16: 91-100.

CARMO FILHO F; OLIVEIRA OF. 1989. Mossoró: um município do semi-árido nordestino - características e aspectos florísticos. Mossoró: ESAM. 62p. (Coleção Mossoroense, B, 672).

CHAVES SW; NEGREIROS MZ; NOGUEIRA ICC; PEDROSA JF; BEZERRA NETO F; PEREIRA FHF. 2004. Densidade de plantio na produção e qualidade de frutos de melão. Caatinga 17: 39-45.

DIAS RCS; COSTA ND; SILVA PCG; QUEIRÓZ MA; ZUZA F; LEITE LAS; PESSOA PFAP; TARAO DA. 1998. Cadeia produtiva do melão no Nordeste. In: CASTRO AMG; LIMA SMV; GOEDART WJ; FREITAS FILHO A; VASCONCELOS JRP. (eds). Cadeias produtivas e sistemas naturais: prospecção tecnológica. Brasília: EMBRAPA-DPD/ EMBRAPA-SPI. p. 440-493.
FERREIRA DF. 2000. Análises estatísticas por meio do Sisvar para Windows Versão 4.0. In: REUNIÃO ANUAL DA REGIÃO BRASILEIRA DA SOCIEDADE INTERNACIONAL DE BIOMETRIA, 45. Anais ... São Carlos: UFSCar. p. 255-258.

GRANGEIRO LC; PEDROSA JF; BEZERRA NETO F; NEGREIROS MZ. 1999a. Rendimento de híbridos de melão amarelo em diferentes densidades de plantio. Horticultura Brasileira 17: 200-206.

GRANGEIRO LC; PEDROSA JF; BEZERRA NETO F; NEGREIROS MZ. 1999b. Qualidade de híbridos de melão amarelo em diferentes densidades de plantio. Horticultura Brasileira 17: 110-113.

HOLIDAY R. 1969. Plant population and crop yield. Nature 186: 22-24.

IBGE - Instituto Brasileiro de Geografia e Estatística. 2004, 21 de dezembro. Produção agrícola. Disponível em http:// www.ibge.gov.br/.

MENEZES JB, GOMES JÚNIOR J; ARAÚJO NETO SE; SIMÕES AN. 2001. Armazenamento de dois genótipos de melão amarelo sob condições de ambiente. Horticultura Brasileira 19: 42-49.

NUNES GHS; SANTOS JÚNIOR JJS; VALE FA; BEZERRA NETO F; ALMEIDA, AHB; MEDEIROS DC. 2004. Aspectos produtivos e de qualidade de híbridos de melão cultivados no agropolo Mossoró-Assu. Horticultura Brasileira 22: 744-747.

NUNES GHS; SANTOS JÚNIOR JJ; ANDRADE FV; BEZERRA NETO F; MENEZES JB; PEREIRA EWL. 2005. Desempenho de híbridos do grupo inodorus em Mossoró. Horticultura Brasileira 23: 90-94.
PARIS HS; NERSON H; BRUGER Y; EDELSTEIN M; KARCHI Z. 1988. Synchrony of yield of melons as affected by plant type and density. Journal of Horticulture Science 63: 141-147.

PEREIRA FHF; NOGUEIRA ICC; PEDROSA JF; NEGREIROS MZ; BEZERRA NETO F. 2003. Poda da haste principal e densidade de cultivo na produção e qualidade de frutos em híbridos de melão. Horticultura Brasileira 21: 191-196.

RESENDE GM; COSTA ND. 2003. Produção e qualidade do melão em diferentes densidades de plantio. Horticultura Brasileira 21: 690694.

ROBINSON RW; DECKER-WALTERS DS. 1997. Cucurbits. New York: CAB International, 226p.

SALES JÚNIOR R; SOARES SPF; AMARO FILHO J; NUNES GHS; MIRANDA VS. 2004. Qualidade do melão exportado pelo porto de Natal. Horticultura Brasileira 22: 98100.

SILVA PSL; FONSECA JR; MOTA JCA; SILVA J. 2003. Densidades de plantio e rendimento de frutos do meloeiro (Cucumis melo). Revista Brasileira de Fruticultura 25: 245-247. 